\title{
Osteoinduction and Osteoconduction with Porous Beta-Tricalcium Phosphate Implanted after Fibular Resection in Humans
}

\author{
Takashi Ariizumi ${ }^{1}$, Hiroyuki Kawashima1 ${ }^{*}$, Hiroshi Hatano ${ }^{2}$, Tetsuro Yamagishi', Naoki Oike ${ }^{1}$, \\ Taro Sasaki ${ }^{2}$, Hajime Umezu ${ }^{3}$, Yongjun $\mathrm{Xu}^{4}$, Naoto Endo ${ }^{1}$, Akira Ogose ${ }^{5}$
}

\author{
${ }^{1}$ Division of Orthopedic Surgery, Niigata University Graduate School of Medical and Dental Sciences, Niigata, Japan \\ ${ }^{2}$ Department of Orthopedic Surgery, Niigata Cancer Center Hospital, Niigata, Japan \\ ${ }^{3}$ Division of Pathology, Niigata University Hospital, Niigata, Japan \\ ${ }^{4}$ Department of Orthopedic Surgery, Harbin Medical University Hospital, Harbin, China \\ ${ }^{5}$ Department of Orthopedic Surgery, Uonuma Kikan Hospital, Niigata, Japan \\ Email: ariiz075@yahoo.co.jp, *inskawa@med.niigata-u.ac.jp
}

How to cite this paper: Ariizumi, T., Kawashima, H., Hatano, H., Yamagishi, T., Oike, N., Sasaki, T., Umezu, H., Xu, Y.J., Endo, N. and Ogose, A. (2019) Osteoinduction and Osteoconduction with Porous Beta-Tricalcium Phosphate Implanted after Fibular Resection in Humans. Journal of Biomaterials and Nanobiotechnology, 10, 159-173.

https://doi.org/10.4236/jbnb.2019.103009

Received: June 3, 2019

Accepted: July 12, 2019

Published: July 15, 2019

Copyright () 2019 by author(s) and Scientific Research Publishing Inc. This work is licensed under the Creative Commons Attribution International License (CC BY 4.0).

http://creativecommons.org/licenses/by/4.0/

c) (†) Open Access

\begin{abstract}
Osteoinductive properties of $\beta$-TCP remain unknown in humans. It is important to improve the bone grafts which have been the standard treatment for bone defect due to their biocompatibility and bone-healing properties. The purpose of this study was to radiologically clarify the bone forming property of $\beta$-TCP by evaluating the replacement of $\beta$-TCP by newly formed bone in the defect after fibular resection and to examine the histological features of a $\beta$-TCP specimen three months after grafting. Radiographs of 17 patients who underwent $\beta$-TCP grafting were evaluated. Osteoinductive and osteoconductive properties were assessed by examining bone formation from the remnant fibula, periosteum, and $\beta$-TCP alone. In one case, $\beta$-TCP was removed later because of postoperative complications and was evaluated histologically. Twenty two of 34 sites between the remnant fibula and $\beta$-TCP had achieved good bone regeneration. Five of 14 sites between the periosteum and $\beta$-TCP had achieved good bone regeneration. We found immature but evident bone formation in three cases with no osseous and periosteal sites. Histological analysis revealed bone formation on the outer macropore surface of $\beta$-TCP. Some blood vessels formed in the macropores expressed CD31 and CD34, while a few lymphatic vessels expressed CD34 and podoplanin. Thus, the osteoinductive ability of $\beta$-TCP alone was demonstrated in humans radiographically for the first time. The histological morphology of $\beta$-TCP was demonstrated at an early stage after grafting in humans.
\end{abstract}

\section{Keywords}

Osteoinduction, Osteoconduction, Beta-Tricalcium Phosphate, Bioactive 


\section{Introduction}

Recently, bioactive ceramics have gained popularity for filling bone defect secondary to trauma or tumor resection [1] [2] [3] [4] [5]. Autogenous bone grafts have been the standard treatment for bone loss due to their biocompatibility and bone-healing properties [6]. However, the amount of bone that can be harvested from a patient's bone is a limitation. Therefore, bioactive ceramic substitutes are a suitable option for filling large bone defects. A variety of synthetic ceramic substitutes have been developed to fill bone defects [7]. Hydroxyapatite $\left(\mathrm{Ca}_{10}\left(\mathrm{PO}_{4}\right)_{6}(\mathrm{OH})_{2}\right)$, beta-tricalcium phosphate $\left(\beta\right.$-TCP) $\left(\mathrm{Ca}_{3}\left(\mathrm{PO}_{4}\right)_{2}\right)$, and their derivatives and combinations are the most commonly used ceramic materials in bone surgeries [8].

Previously, we reported that $\beta$-TCP is a suitable bone-filling agent in clinical applications [3] [4] [5] [9] [10] [11]. Beta-TCP has been shown to have good biocompatibility and osteoconductivity in both animal experiments and clinical settings [12] [13]. Numerous basic studies have demonstrated that $\beta$-TCP enables good osteoconduction in various animals including human bone [3] [9] [13] [14]. However, calcium phosphate ceramics generally lack the osteoinductive properties needed for bone healing in large defect [15]. It has been shown that certain porous calcium phosphate bioceramics and coatings on metal implants induce ectopic bone formation when implanted in the muscles of large animals without adding osteogenic cells or bone growth factors prior to implantation [16] [17] [18] [19] [20], while the osteoinductive properties of $\beta$-TCP remain unknown in humans.

Meanwhile, fibular bone defects are sometimes seen in surgeries for tumors of bones and soft tissues because of fibular bone grafts or resections of the primary fibular tumor. Before 2004, fibular defects were not reconstructed in our institutions. On the other hand, the residual functional deficit or ankle instability had been reported following fibulectomy [21] [22]. Since 2005, highly purified $\beta$-TCP blocks have been used to fill bone defects after resection of the fibula. The purpose of this study was to radiologically clarify the osteoinductive and osteoconductive properties of $\beta$-TCP in humans by evaluating the newly formed bone in the donor sites following the use of $\beta$-TCP.

Additionally, reports of histological examination of $\beta$-TCP in human bones at early stage are rare. The secondary purpose of this study was to examine the histological features of a $\beta$-TCP specimen three months after grafting.

\section{Patients, Materials and Methods}

Between 2005-2014, 17 patients who required $\beta$-TCP grafts after fibular resection at the Division of Orthopedic Surgery, Niigata University Graduate School of Medical and Dental Sciences, and the Department of Orthopedic Surgery, 
Niigata Cancer Center Hospital were included in this study. We reviewed their clinical findings and radiographs. This study was approved by the research ethics committee of Niigata University Graduate School of Medical and Dental Sciences and Niigata Cancer Center Hospital (Approval no. 2017-0099). We obtained informed consent from each patient. We have used highly purified $\beta$-TCP since 1999 to fill bone defects. Beta-TCP (OSferion ${ }^{\circledR}$ Olympus, Tokyo Japan) was manufactured in an extraordinary high purity [3] [4] [5].

The primary objective was to radiographically evaluate the osteoconductive and osteoinductive properties of $\beta$-TCP in humans. In all patients, a plain anteroposterior radiograph of the lower leg was obtained. The immediate postoperative and the last follow-up X-rays were assessed to evaluated the bone formation of $\beta$-TCP. Three radiographic points were established in order to evaluate the osteoconductive and osteoinductive properties of the grafted $\beta$-TCP (Figure 1). The first point was defined by bone formation between the remnant fibula and $\beta$-TCP regardless of periosteal preservation, which was called osteogenesis from the remnant fibula. The second point, called osteogenesis from the periosteum, was defined as bone formation between the periosteum and $\beta$-TCP in cases with periosteal preservation, such as non-vascularized free fibular grafts. In this way, we evaluated the osteoconductive properties of $\beta$-TCP scaffolds to investigate directing bone formation at local osseous sites. The third point was defined by bone formation using $\beta$-TCP scaffolds alone in cases with fibular resection along

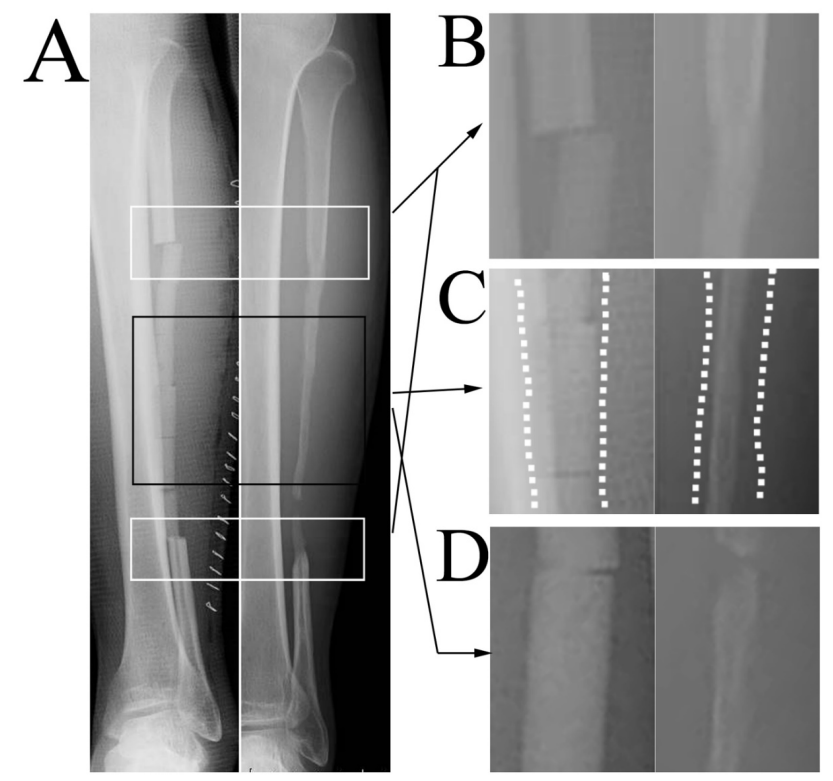

Figure 1. Schematic diagram of radiographic assessments. (A) The full length of the fibula in the immediately post-operative period (left) and at the last follow-up (right); (B) Bone formation between the remnant fibula and $\beta$-TCP, which was called osteogenesis from the remnant fibula; (C) Bone formation between the periosteum (dotted line) and $\beta$-TCP in cases with periosteal preservation such as non-vascularized free fibular grafts, which was called osteogenesis from the periosteum; (D) Bone formation using $\beta$-TCP scaffolds alone in cases with vascularized fibular grafts and no periosteum at the donor site, which was called osteogenesis induced by $\beta$-TCP alone. 
with the periosteum such as vascularized fibular grafts, which was called osteogenesis induced by $\beta$-TCP alone. In this way, we evaluated its osteoinductive properties. Based on the radiographic findings at the last follow-up, the newly formed bone could be divided into three categories: poor (little or no bone formation at the donor site), fair (some discontinuous or immature bone formation), and good (regeneration of cortical bone with a medullary cavity) (Table 1).

The secondary objective was to histologically evaluate the early osteoconductive ability of $\beta$-TCP in humans. This was evaluated in one patient who underwent non-vascularized free fibular graft. It was an 18-year-old male with osteosarcoma arising from the left distal femur (Case 7). After the preoperative chemotherapy, we performed wide resection and reconstruction using irradiated bone and ipsilateral non-vascularized free fibular graft. Beta-TCP blocks were placed at the donor site. Three months later, surgical site infection occurred in the distal femur, and the left thigh was amputated. The $\beta$-TCP specimen was removed from the amputated leg after obtaining informed consent. This specimen was immersed in $4 \%$ paraformaldehyde for 5 - 7 days, decalcified with ethylenediaminetetraacetic acid, and embedded with paraffin. Hematoxylin and eosin staining was performed to detect the newly formed bone. To detect osteoclasts, TRAP staining was performed according to Burstone's Azo dye method [23] with some modifications [24]. To detect the morphological appearance of $\beta$-TCP, immunohistochemical staining was performed with the following primary antibodies: CD68 (pan-macrophage marker) (KP-1; Dako), CD31 (vascular endothelium marker) (Dako), CD34 (endothelium marker) (Dako), and podoplanin (D2-40; lymphatic endothelial marker) (Dako). This specimen was incubated with these primary antibodies and reacted with universal immuno-peroxidase polymer, anti-mouse and anti-rabbit antibodies (Histofine Simple Stain MAX PO (MULTI); Nichirei, Tokyo, Japan). The peroxidase activity was detected using 3'-diaminobenzidine tetrahydrochloride (Nichirei). The specimen was counter-stained with hematoxylin.

\section{Results}

\subsection{Clinical Data of the Patients}

The demographic data is presented in Table 2. Eight patients were males and nine females. The mean age was 27.2 years (range, 9 - 72 years). The mean follow-up period postoperatively was 56.8 months (range, 2.4 - 179 months). Fourteen

Table 1. Scoring of the radiographic evaluation.

\begin{tabular}{cc}
\hline Score & Radiographic evaluation \\
\hline Poor & Little or no bone formation at the donor site \\
Fair & Some discontinuous or immature bone formation \\
Good & Regeneration of cortical bone with a medullary cavity
\end{tabular}


Table 2. Summary of the patients.

\begin{tabular}{|c|c|c|c|c|c|c|c|c|c|c|}
\hline $\begin{array}{l}\text { Case } \\
\text { No. }\end{array}$ & Age & Sex & Diagnosis & $\begin{array}{l}\text { Follow-up } \\
\text { period } \\
\text { (Months) }\end{array}$ & $\begin{array}{l}\text { Surgical procedure } \\
\text { of the primary site }\end{array}$ & $\begin{array}{l}\text { Resection } \\
\text { length of the } \\
\text { fibula }(\mathrm{mm})\end{array}$ & $\begin{array}{l}\text { Amount of } \\
\text { the grafted } \\
\beta \text {-TCP }(\mathrm{g})\end{array}$ & $\begin{array}{l}\text { Condition of } \\
\text { the periosteum } \\
\text { at the donor } \\
\text { site }\end{array}$ & $\begin{array}{l}\text { Infiltration } \\
\text { of bone } \\
\text { marrow } \\
\text { aspirates }\end{array}$ & $\begin{array}{l}\text { Postoperative } \\
\text { symptom }\end{array}$ \\
\hline 1 & 12 & $\mathrm{~F}$ & $\begin{array}{l}\text { Osteoid osteoma, } \\
\text { rt. fibula }\end{array}$ & 23.8 & Wide resection & 71 & 2.5 & Excised & yes & no \\
\hline 2 & 12 & $\mathrm{~F}$ & CS, rt. femur & 178 & Wide resection, VFG & 188 & 14.4 & Excised & no & no \\
\hline 3 & 13 & $\mathrm{~F}$ & OS, lt. radius & 106 & Wide resection, VFG & 233 & 5.4 & Excised & yes & no \\
\hline 4 & 21 & $\mathrm{M}$ & $\mathrm{ABC}$, lt. femur & 2.36 & Curettage, NVFG & 101 & 2.3 & Preserved & no & no \\
\hline 5 & 54 & $\mathrm{~F}$ & GCTB, lt. tibia & 59.2 & Curettage, NVFG & 104 & 3.5 & Preserved & no & no \\
\hline 6 & 55 & $\mathrm{~F}$ & GCTB, lt. ischium & 10.8 & Wide resection, NVFG & 160 & 3.9 & Preserved & no & no \\
\hline 7 & 18 & M & OS, rt. femur & 3.18 & $\begin{array}{l}\text { Wide resection, NVFG, } \\
\text { Irradiated bone graft }\end{array}$ & 219 & 7.3 & Preserved & no & no \\
\hline 8 & 72 & $\mathrm{~F}$ & UPS, lt. upper arm & 32.2 & Wide resection, NVFG & 132 & 10.4 & Preserved & no & no \\
\hline 9 & 28 & $\mathrm{~F}$ & FD, lt. femur & 20.1 & Curettage, NVFG & 104 & 3.5 & Preserved & no & no \\
\hline 10 & 21 & $\mathrm{~F}$ & SBC, rt. humerus & 13.8 & Curettage, NVFG & 170 & 13.1 & Preserved & no & no \\
\hline 11 & 58 & $\mathrm{M}$ & $\mathrm{CB}$, rt. femur & 13.5 & Curettage, NVFG & 180 & 13.1 & Preserved & no & no \\
\hline 12 & 20 & $\mathrm{M}$ & GCTB, lt. femur & 17.8 & Curettage, NVFG & 133 & 4.6 & Preserved & no & no \\
\hline 13 & 29 & $\mathrm{M}$ & $\mathrm{FD}$, rt. femur & 69.3 & Curettage, NVFG & 106 & 3.5 & Preserved & no & Ankle pain \\
\hline 14 & 14 & $\mathrm{M}$ & OS, lt. femur & 130 & $\begin{array}{c}\text { Wide resection, prosthetic } \\
\text { reconstruction augmented } \\
\text { with NVFG }\end{array}$ & 133 & 3.1 & Preserved & no & no \\
\hline 15 & 16 & $\mathrm{~F}$ & SBC, rt. femur & 65.4 & Curettage, NVFG & 90 & 3.1 & Preserved & no & no \\
\hline 16 & 10 & $\mathrm{M}$ & FD, lt. femur & 168 & Curettage, NVFG & 110 & 3.1 & Preserved & no & no \\
\hline 17 & 9 & $\mathrm{~F}$ & $\begin{array}{c}\text { Histiocytic sarcoma, } \\
\text { lt. thigh }\end{array}$ & 51.6 & $\begin{array}{l}\text { Wide resection, NVFG, } \\
\text { Irradiated bone graft }\end{array}$ & 126 & 4.2 & Preserved & no & no \\
\hline
\end{tabular}

F, Female; M, Male; rt., right; lt., left; CS, Chondrosarcoma; OS, Osteosarcoma; ABC, Aneurysmal bone cyst; GCTB, Giant cell tumor of bone; UPS, Undifferentiated pleomorphic sarcoma; FD, Fibrous dysplasia; SBC, Solitary bone cyst; VFG, Vascularized fibular graft; NVFG, Non-vascularized fibular graft.

of 17 patients underwent resection of the non-vascularized fibulas with preservation of the periosteum at the donor site. Two patients underwent resection of vascularized fibulas and only one underwent resection of the fibula with a wide margin due to primary bone tumor. The periosteum was not preserved in these 3 cases. In two patients, the bone marrows were obtained by aspiration from the iliac bone and were infiltrated with $\beta$-TCP blocks before implantation to stimulate bone formation. No complications were observed at the site of fibular defects. One patient (Case 13) complained of ankle pain at the last follow-up, though it is unclear if it was related to the implantation of $\beta$-TCP blocks.

\subsection{Radiographic Assessment of $\beta$-TCP}

We assessed the three radiographic points mentioned previously (Table 3). The degree of osteogenesis from the remnant fibula was assessed at 34 sites between the remnant fibula and $\beta$-TCP (sum of proximal and distal remnants of the fibula). 
The results noted were as follows: 22 (64.7\%), good; 0, fair; and 12 (35.3\%), poor. At 14 sites between the periosteum and $\beta$-TCP, the degree of osteogenesis from the periosteum was assessed, which had the following results: 5 (35.7\%), good; 5 (35.7\%), fair; and 4 (28.6\%), poor. Good results in both these radiographic points were noted in case 17 (Figure 2), which revealed complete regeneration of the fibula radiologically. Figure 3 describes the case with poor osteogenesis from the remnant fibula (case 9). This case had absorption of the proximal and distal sides of the $\beta$-TCP blocks. In three patients who underwent resection of the fibula with periosteum, the degree of osteogenesis induced by $\beta$-TCP alone was assessed and the results were as follows: 0, good; 2 (66.7\%), fair; and 1 (33.3\%), poor. For example, newly formed bone with a medullary cavity was observed from the remnant proximal and distal fibula in case 1 (Figure 4). A little bone formation was

Table 3. The result of the radiographic assessment.

\begin{tabular}{|c|c|c|c|c|}
\hline & Total & Poor & Fair & Good \\
\hline & & \multicolumn{3}{|c|}{ Mean follow-up period (range), month } \\
\hline \multirow{2}{*}{$\begin{array}{l}\text { Osteogenesis from } \\
\text { the remnant fibula }\end{array}$} & 34 & 12 & 0 & 22 \\
\hline & & $20.4(2.4-106.3)$ & 0 & $76.7(106.3-178.8)$ \\
\hline \multirow{2}{*}{$\begin{array}{l}\text { Osteogenesis from } \\
\text { the periosteum }\end{array}$} & 14 & 4 & 5 & 5 \\
\hline & & $18.9(2.4-59.2)$ & $19.5(13.5-32.2)$ & $96.8(51.6-167.8)$ \\
\hline \multirow{2}{*}{$\begin{array}{l}\text { Osteogenesis induced } \\
\text { by } \beta \text {-TCP alone }\end{array}$} & 3 & 1 & 2 & 0 \\
\hline & & 23.8 & $142.6(106.3-178.8)$ & 0 \\
\hline
\end{tabular}
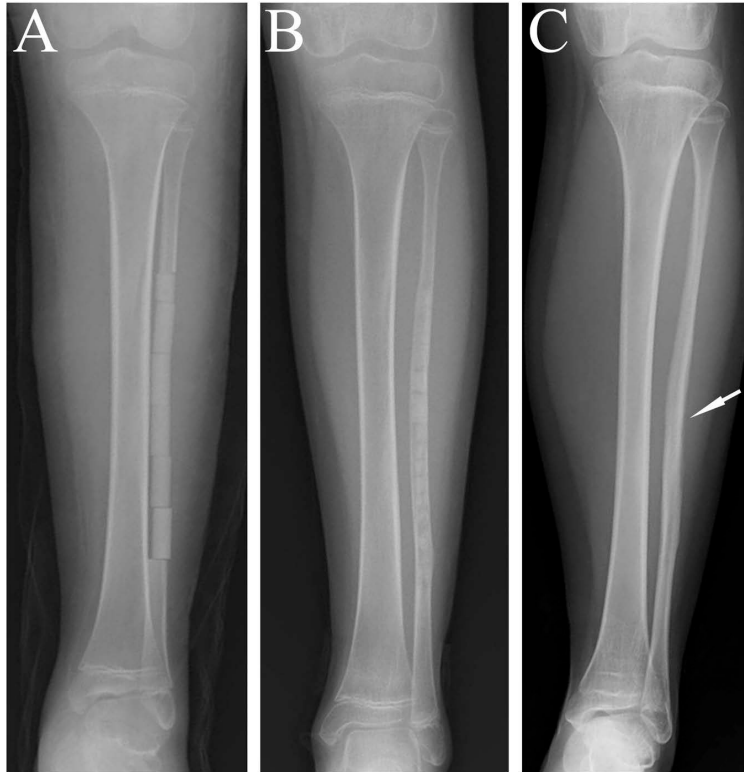

Figure 2. Radiographs of a 9-year-old female with histiocytic sarcoma (Case 17; Periosteum preserved). (A) Immediate post-operative radiograph demonstrating the $\beta$-TCP blocks filled in the bone defect; (B) Five months later, $\beta$-TCP blocks were unclear and bridging callus was formed from top to bottom at the donor site; (C) Fifty-two months later, complete regeneration of the fibula was attained (arrow). 

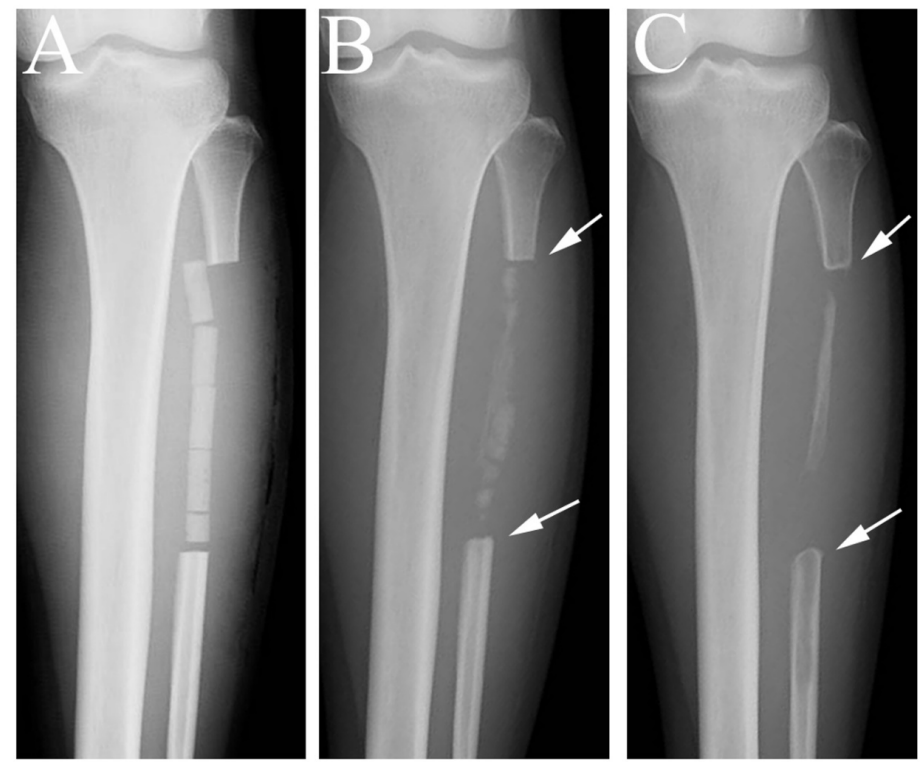

Figure 3. Radiographs of a 28-year-old female with fibrous dysplasia (Case 9; Periosteum preserved). (A) Immediate post-operative radiograph demonstrating the $\beta$-TCP blocks filled in the bone defect; (B) Four months later, the middle part of the $\beta$-TCP blocks were unclear and bridging callus was formed but the proximal and distal sides of the $\beta$-TCP blocks were absorbed (arrow); (C) Nineteen months later, the proximal and distal side of the $\beta$-TCP blocks were absent and newly formed bone was not observed (arrow). Immature bone was present in the central part at the donor site.
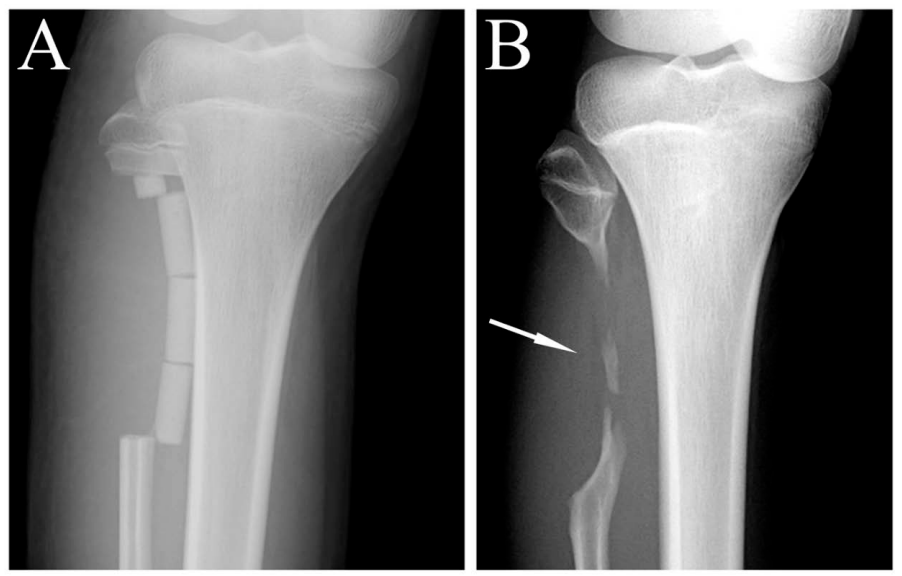

Figure 4. Radiographs of a 12-year-old female with osteoid osteoma (Case 1; Periosteum excised). (A) The proximal shaft of the fibula was resected with the tumor. The periosteum was removed with the tumor. Immediate postoperative radiograph demonstrating some $\beta$-TCP blocks filled in the bone defect. Bone marrow aspirates were infiltrated with $\beta$-TCP blocks before implantation; (B) Twenty-one months later, $\beta$-TCP blocks were absent. Newly formed bone was observed from the remnant fibula (arrow), while little bone was present in the central part at the donor site. Good results in the degree of osteogenesis from the remnant fibula were noted at the proximal and distal remnant fibula.

observed within the central part of the bone defect. Bone marrow aspirates were infiltrated with $\beta$-TCP blocks before implantation in this case. Poor osteogenesis induced by $\beta$-TCP alone was noted. In case 3 , a regenerated medullary cavity 
was observed. However, bone regeneration was immature and discontinuous in the central part of the bone defect (Figure 5). Bone marrow aspirates were also infiltrated with $\beta$-TCP blocks before implantation in this case. Fair osteogenesis induced by $\beta$-TCP alone was noted. In case 2 , almost all of the grafted $\beta$-TCP was absorbed and immature regeneration of the fibular bone was observed (Figure 6). Fair osteogenesis induced by $\beta$-TCP alone was noted. However, bone regeneration was evident in the central part of the bone defect although bone marrow aspirates were not infiltrated with $\beta$-TCP blocks before implantation in this case.

\subsection{Histological Evaluation}

The specimen was evaluated on an axial section (Figure 7). The section with hematoxylin and eosin staining showed fibrovascular invasion into the macropores of $\beta$-TCP in the central region of the implanted area as well as in the peripheral region. There was no evidence of any newly formed bone in the central region, while abundant new bone formation on $\beta$-TCP was observed in the peripheral region. The cuboidal and columnar shaped osteoblasts lined the surface of the newly formed bone. In the middle region, numerous multinucleated-giant cells (probably osteoclasts) were attached to $\beta$-TCP. These cells were strongly positive for TRAP and CD68 (Figure 8). CD68 positive cells were scarce and TRAP positive cells were absent in the center region. Some vessels were formed
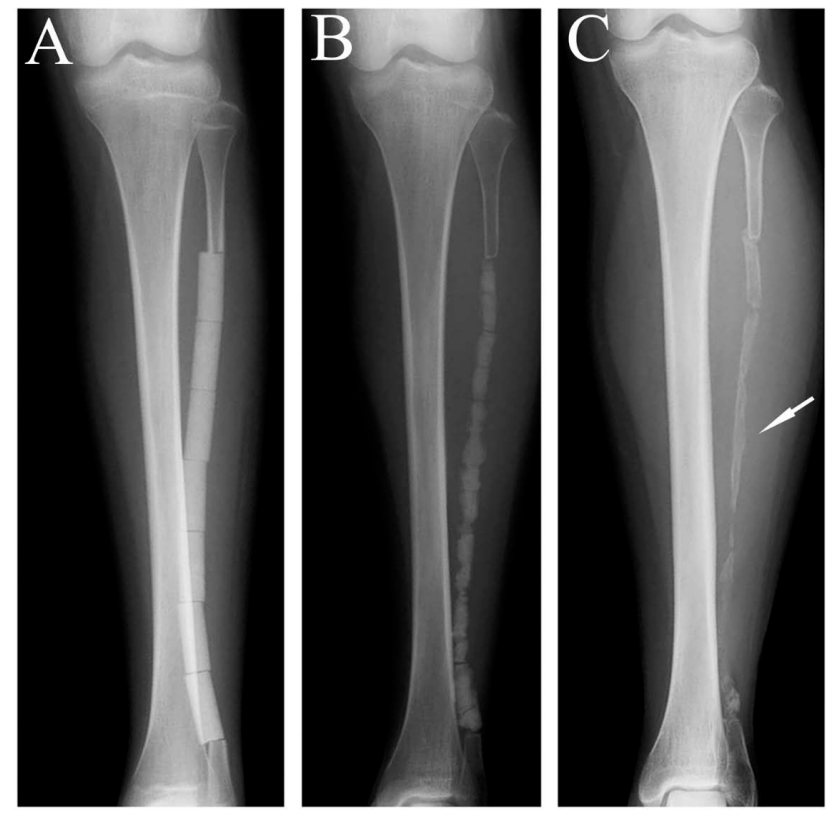

Figure 5. Radiographs of a 13-year-old female with osteosarcoma (Case 3; Periosteum excised). (A) Immediate postoperative radiograph demonstrating some $\beta$-TCP blocks filled in the bone defect. Bone marrow aspirates were infiltrated with $\beta$-TCP blocks before implantation; (B) Sixteen months later, $\beta$-TCP blocks were partially absorbed and bone formation was not clear; $(C)$ One hundred and thirty-two months later, a medullary cavity was observed but bone regeneration was immature (arrow) in the central part at the donor site. The $\beta$-TCP blocks adjacent to the remnant fibulas had regenerated to bone. 


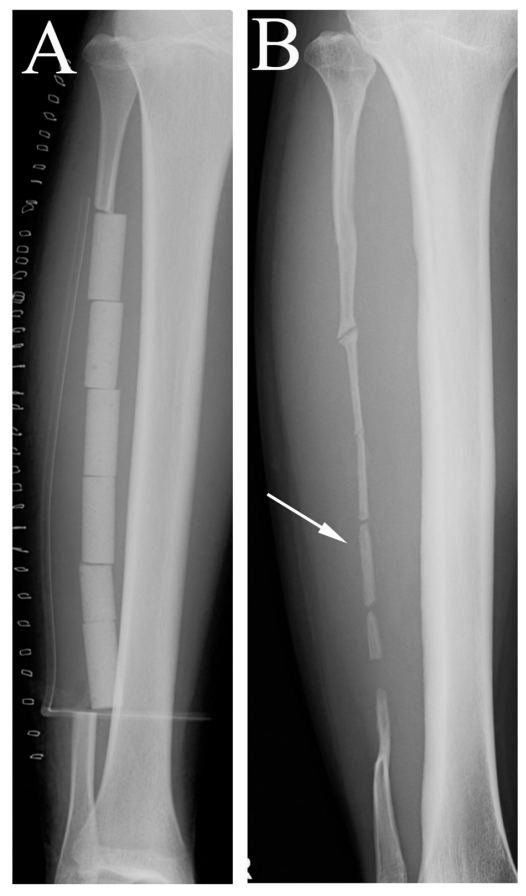

Figure 6. Radiographs of a 12-year-old female with chondrosarcoma (Case 2; Periosteum excised). (A) Immediate post-operative radiograph demonstrating $\beta$-TCP blocks filled in the bone defect. Bone marrow aspirates were not infiltrated with $\beta$-TCP blocks before implantation; (B) One hundred and seventy-nine months later, almost all of the grafted $\beta$-TCP was absorbed and the regeneration of fibular bone was observed with bone marrow cavity, although the bone formation was immature.
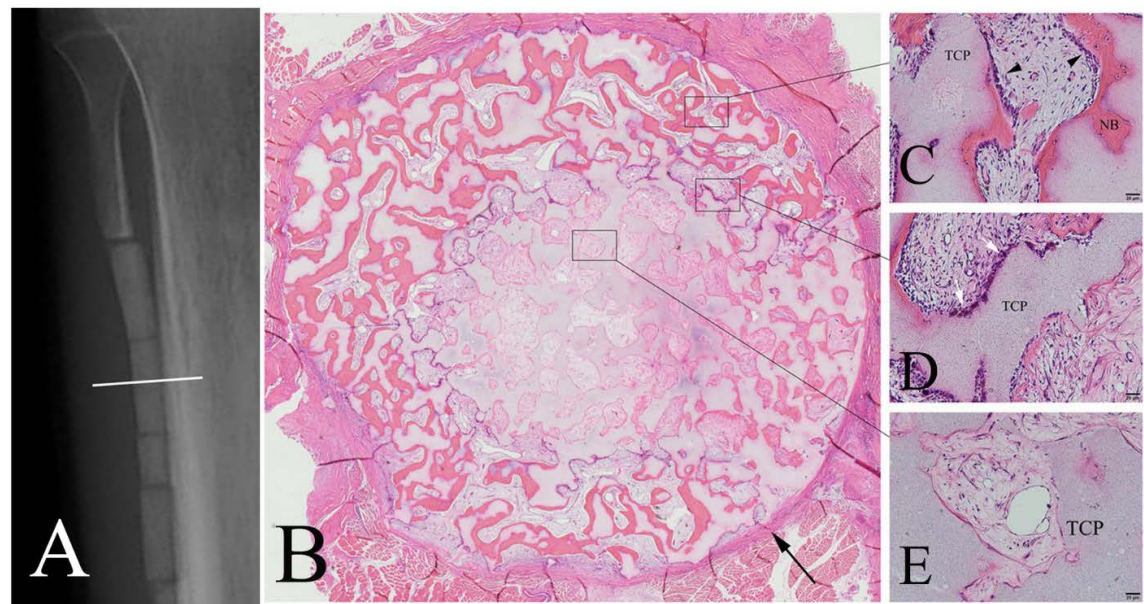

Figure 7. Histological sections stained with hematoxylin and eosin in the $\beta$-TCP specimen. Three months after the surgery, the $\beta$-TCP specimen was removed and the axial section was used for the histological assessment (A). The periosteum was preserved circumferentially (B, black arrow). Fibrovascular invasion was seen in the macropores of $\beta$-TCP in the central region of the implanted area (E) as well as in the peripheral region. Abundant new bone formation on $\beta$-TCP was observed in the peripheral region. The cuboidal and columnar shaped cells (probably osteoblasts) lined the surface of the newly formed bone ( $\mathrm{C}$, black arrowheads). Between the peripheral abundant bone and central scarce bone, numerous multinucleated-giant cells (probably osteoclasts) were attached to $\beta$-TCP (D, white arrows). TCP, $\beta$-tricalcium phosphate; NB, newly formed bone. 


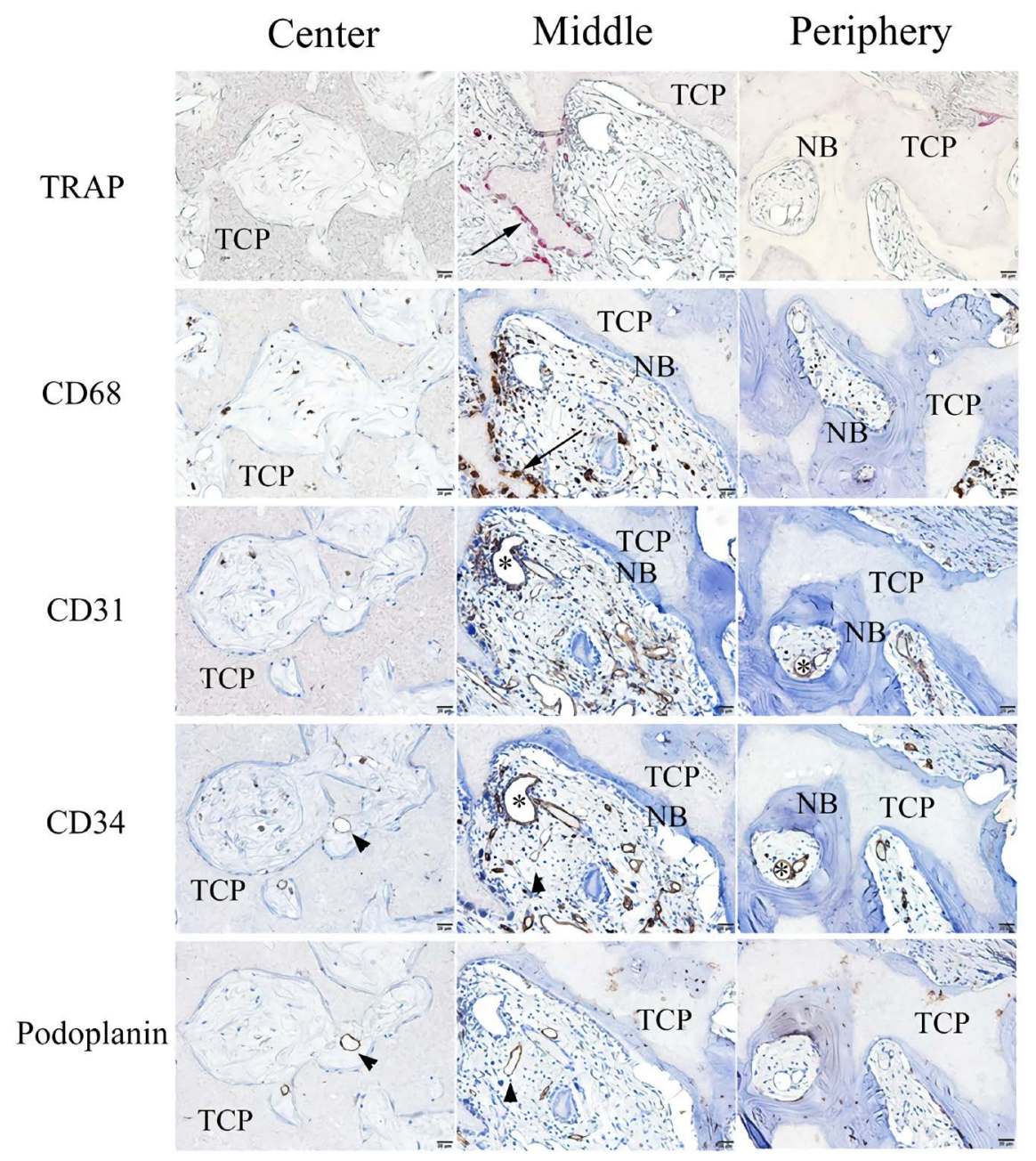

Figure 8. TRAP and immunohistochemical staining analysis of the $\beta$-TCP specimen in case 7. Numerous multinucleated-giant cells attached to $\beta$-TCP were strongly positive for TRAP and CD68. The TRAP and CD68 positive cells were mostly located in the middle region in a serial section (arrows). CD68 positive cells were scarce and TRAP positive cells were absent in the central region. Some vessels were found in every region of the specimen, although these vessels were mainly concentrated in the middle region. Most vessels in the macropores expressed CD31 and CD34 in a serial section (asterisks), while few capillaries expressed CD34 and podoplanin (arrowheads). TCP, $\beta$-tricalcium phosphate; NB, newly formed bone.

in the macropores of $\beta$-TCP. These vessels were found in every region of the specimen, although they were mainly concentrated in the middle region. Most of these vessels expressed CD31 and CD34 on a serial section, while fewer vessels expressed CD34 and podoplanin.

\section{Discussion}

The primary aim of this study was to clarify the osteoconductive and osteoinductive properties of $\beta$-TCP in human radiologically. We found satisfactory bone formation between the remnant fibula and $\beta$-TCP, and between the periosteum and $\beta$-TCP. The findings corroborated with those of previous studies 
that documented the regeneration of the fibula with $\beta$-TCP [25]. We evaluated the osteoconductive properties of $\beta$-TCP using two radiographic points. The osteogenesis from the remnant fibula was good in $22(64.7 \%)$ patients but poor in 12 (35.3\%). In most cases with good results, almost normal cortical bone and intramedullary space was observed in the places of $\beta$-TCP blocks. These findings suggest the process of bony ingrowth from the margins of remnant fibula over the $\beta$-TCP block that can be resorbed. The probable reason for the poor results may be the short follow-up periods, which were due to dropping out of the patients or death due to underlying diseases. There were 5 (35.7\%), 5 (35.7\%), and $4(28.6 \%)$ results of good, fair, and poor osteogenesis from the periosteum. The periosteum is known to be pluripotent. It consists of osteoblastic and chondrogenic cells and it may be utilized to engineer new bone formation in vivo [26]. Autogenic periosteum could increase the bioactivity of ceramics in heterosites and improve bone formation in porous calcium phosphate ceramics [27]. The evaluation of osteogenesis from the periosteum suggested that the combination of $\beta$-TCP and osteoblastic cells in the periosteum accelerate bone formation. These two findings demonstrated that $\beta$-TCP has good osteoconductive properties in humans.

The result of osteogenesis induced by $\beta$-TCP alone demonstrated that newly formed bone was observed in three cases although the bone formation was immature. In two cases, the bone marrow stromal cells might have promoted bone formation because bone marrow aspirates were infiltrated with $\beta$-TCP blocks before implantation. While in one case, new bone formation was observed although there was no periosteum at the donor site and bone marrow aspirates were not infiltrated. This finding suggested that $\beta$-TCP had osteoinductive properties in humans with no additional agents required. Arai et al. reported good osteoconductive properties of $\beta$-TCP radiographically. However, new bone was not observed at the donor site with $\beta$-TCP in the case of patients who underwent vascularized fibular grafting [25]. In the past, osteoinduction of $\beta$-TCP without concomitant use of bone marrow cells or bone-inductive cytokines has not been reported in humans. On the other hand, porous $\beta$-TCP has been known to demonstrate osteoinductive ability in certain animals [15]-[20]. We previously investigated the process of osteoinduction in porous $\beta$-TCP in canine dorsal muscles, which suggested that the micropores on the macropore surfaces are critical for this process [10] [11]. The result suggests that the microstructure of porous $\beta$-TCP is also an important factor in the process of osteoinduction in humans. The current study is noteworthy because the osteoinductive ability of $\beta$-TCP alone was demonstrated in humans radiographically for the first time.

The secondary aim was histological examination of a $\beta$-TCP specimen in human bone. The findings demonstrated the promotion of bone formation in the axial section of the specimen. Abundant new bone formation on $\beta$-TCP was observed in the peripheral region. In this case, the periosteum was preserved at the donor site. This result suggested that the osteoblasts of the periosteum infiltrated the macropores of $\beta$-TCP and that the bone formation extended from the peri- 
phery to the center of $\beta$-TCP. In the middle regions, numerous multinucleated-giant cells that were positive for TRAP and CD68 were directly attached to $\beta$-TCP, thus, suggesting that $\beta$-TCP is resorbed by osteoclasts and leads to osteoblastic new bone formation over the surface of $\beta$-TCP. In immunohistochemical analysis, $\mathrm{CD} 31$ and $\mathrm{CD} 34$ positive vessels were generally found in the macropores. CD31 and CD34 are vascular endothelial marker which express in the blood vessels [28]. This finding suggested the importance of blood vessels for bone regeneration. A few vessels expressed CD34 and podoplanin. CD34 is an endothelial marker, while podoplanin is a selective marker of lymphatic endothelium [29]. This finding suggested that lymphatic vessels invaded the macropores of $\beta$-TCP along with the blood vessels. Edwards et al. reported that lymphatic vessels are absent in normal bone but are observed in pathological conditions such as lymphangioma, some primary bone tumors, and Gorham-Stout disease [30]. However, lymphatic vessels in the regenerated bone from $\beta$-TCP have not been previously reported. The current study is noteworthy because it is the first to report lymphatic vessels in $\beta$-TCP histologically, although the role was not clear for bone regeneration.

There are several limitations to this study. The number of the patients was small. This study included patients with only short-term follow-up. There were no control patients who underwent fibular resection without grafting of $\beta$-TCP at the donor site. Future studies should include more cases with a longer follow-up to corroborate these findings.

In this study, we demonstrated radiographic regeneration of bone in place of $\beta$-TCP and histological morphology of $\beta$-TCP in the early stages following grafting in humans. Beta-TCP can be an excellent bone substitute to augment the formation of new bone in clinical use. The bone forming process of $\beta$-TCP in humans needs to be evaluated in future studies. Further studies are needed to demonstrate the osteoinductive properties of $\beta$-TCP in both animal experiments and clinical settings.

\section{Conclusion}

The osteoinductive and osteoconductive properties of $\beta$-TCP were radiologically demonstrated by evaluating the replacement of $\beta$-TCP by newly formed bone in fibular defects in humans. Beta-TCP has good osteoconductive and weak osteoinductive properties in humans. Histological examination suggested that the osteoblasts of the periosteum infiltrated the macropores of $\beta$-TCP and the bone formation extended from the periphery to the center of $\beta$-TCP. Beta-TCP is resorbed by osteoclasts and leads to osteoblastic new bone formation over the surface of $\beta$-TCP.

\section{Acknowledgements}

We thank Tetsuo Hotta MD, PhD from the Division of Orthopedic Surgery, Niigata University Graduate School of Medical and Dental Sciences for help and 
advice regarding this manuscript.

\section{Conflicts of Interest}

The authors declare no conflicts of interest regarding the publication of this paper.

\section{References}

[1] Yamamoto, T., Onga, T., Marui, T. and Mizuno, K. (2000) Use of Hydroxyapatite to Fill Cavities after Excision of Benign Bone Tumours. Clinical Results. Journal of Bone and Joint Surgery British, 82, 1117-1120.

[2] Uchida, A., Araki, N., Shinto, Y., Yoshikawa, H., Kurisaki, E. and Ono, K. (1990) The Use of Calcium Hydroxyapatite Ceramic in Bone Tumour Surgery. Journal of Bone and Joint Surgery British, 72, 298-302. https://doi.org/10.1302/0301-620X.72B2.2155908

[3] Ogose, A., Hotta, T., Hatano, H., Kawashima, H., Tokunaga, K., Endo, N. and Umezu, H. (2002) Histological Examination of Beta-Tricalcium Phosphate Graft in Human Femur. Journal of Biomedical Materials Research, 63, 601-604. https://doi.org/10.1002/jbm.10380

[4] Ogose, A., Hotta, T., Kawashima, H., Kondo, N., Gu, W., Kamura, T. and Endo, N. (2005) Comparison of Hydroxyapatite and Beta Tricalcium Phosphate as Bone Substitutes after Excision of Bone Tumors. Journal of Biomedical Materials Research Part B: Applied Biomaterials, 72, 94-101.

https://doi.org/10.1002/jbm.b.30136

[5] Ogose, A., Kondo, N., Umezu, H., Hotta, T., Kawashima, H., Tokunaga, K., Ito, T., Kudo, N., Hoshino, M., Gu, W. and Endo, N. (2006) Histological Assessment in Grafts of Highly Purified Beta-Tricalcium Phosphate (OSferion) in Human Bones. Biomaterials, 27, 1542-1549. https://doi.org/10.1016/j.biomaterials.2005.08.034

[6] Keating, J.F. and McQueen, M.M. (2001) Substitutes for Autologous Bone Graft in Orthopaedic Trauma. Journal of Bone and Joint Surgery British, 83, 3-8.

[7] Giannoudis, P.V., Dinopoulos, H. and Tsiridis, E. (2005) Bone Substitutes: An Update. Injury, 36, S20-S27. https://doi.org/10.1016/j.injury.2005.07.029

[8] Šponer, P., Urban, K., Kučera, T., Kohout, A., Brtková, J. and Knížek, J. (2011) The Use of Interconnected Beta-Tricalcium Phosphate as Bone Substitute after Curettage of Benign Bone Tumours. European Journal of Orthopedic Surgical Traumatology, 21, 235-241. https://doi.org/10.1007/s00590-010-0701-X

[9] Kondo, N., Ogose, A., Tokunaga, K., Ito, T., Arai, K., Kudo, N., Inoue, H., Irie, H. and Endo, N. (2005) Bone Formation and Resorption of Highly Purified Beta-Tricalcium Phosphate in the Rat Femoral Condyle. Biomaterials, 26, 5600-5608. https://doi.org/10.1016/j.biomaterials.2005.02.026

[10] Kondo, N., Ogose, A., Tokunaga, K., Umezu, H., Arai, K., Kudo, N., Hoshino, M., Inoue, H., Irie, H., Kuroda, K., Mera, H. and Endo, N. (2006) Osteoinduction with Highly Purified Beta Tricalcium Phosphate in Dog Dorsal Muscles and the Proliferation of Osteoclasts before Heterotopic Bone Formation. Biomaterials, 27, 4419-4427. https://doi.org/10.1016/j.biomaterials.2006.04.016

[11] Ariizumi, T., Ogose, A., Kondo, N., Kawashima, H., Hotta, T., Kudo, N., Hoshino, M., Inoue, H., Irie, H. and Endo, N. (2013) The Role of Microstructure of Highly Purified Beta-Tricalcium Phosphate for Osteoinduction in Canine Dorsal Muscles. Journal of Biomaterial and Nanobiotechnology, 4, 189-193. 
https://doi.org/10.4236/jbnb.2013.42023

[12] Ozawa, M., Tanaka, K., Morikawa, S., Chazono, M. and Fujii, K. Clinical Study of the Pure Beta-Tricalcium Phosphate-Reports of 167 Cases. Journal of the Eastern Japan Association of Orthopaedics and Traumatology, 12, 409-413.

[13] Chazono, M., Tanaka, T., Komaki, H. and Fujii, K. (2004) Bone Formation and Bioresorption after Implantation of Injectable Beta-Tricalcium Phosphate Granules-Hyaluronate Complex in Rabbit Bone Defects. Journal of Biomedical Materials Research Part A, 70, 542-549. https://doi.org/10.1002/jbm.a.30094

[14] Bodde, E.W., Wolke, J.G., Kowalski, R.S. and Jansen, J.A. (2007) Bone Regeneration of Porous Beta-Tricalcium Phosphate (Conduit ${ }^{\mathrm{TM}} \mathrm{TCP}$ ) and of Biphasic Calcium Phosphate Ceramic (Biosel ${ }^{\circledR}$ ) in Trabecular Defects in Sheep. Journal of Biomedical Materials Research Part A, 82, 711-722. https://doi.org/10.1002/jbm.a.30990

[15] Daculsi, G. and Layrolle, P. (2004) Osteoinductive Properties of Micro Macroporous Biphasic Calcium Phosphate Bioceramics. Key Engineering Materials, 254-256, 1005-1008. https://doi.org/10.4028/www.scientific.net/KEM.254-256.1005

[16] Yamasaki, H. and Sakai, H. (1992) Osteogenic Response to Porous Hydroxyapatite Ceramics under the Skin of Dogs. Biomaterials, 13, 308-312.

https://doi.org/10.1016/0142-9612(92)90054-R

[17] Ripamonti, U. (1991) Bone Induction in Nonhuman Primates. An Experimental Study on the Baboon. Clinical Orthopaedics and Related Research, 269, 284-294. https://doi.org/10.1097/00003086-199108000-00039

[18] Ripamonti, U. (1996) Osteoinduction in Porous Hydroxyapatite Implanted in Heterotopic Sites of Different Animal Models. Biomaterials, 17, 31-35. https://doi.org/10.1016/0142-9612(96)80752-6

[19] Yang, Z., Yuan, H., Tong, W., Zou, P., Chen, W. and Zhang, X. (1996) Osteogenesis in Extraskeletally Implanted Porous Calcium Phosphate Ceramics: Variability among Different Kinds of Animals. Biomaterials, 17, 2131-2137. https://doi.org/10.1016/0142-9612(96)00044-0

[20] Nihouannen, D.L., Daculsi, G., Saffarzadeh, A., Gauthier, O., Delplace, S., Pilet, P. and Layrolle, P. (2005) Ectopic Bone Formation by Microporous Calcium Phosphate Ceramic Particles in Sheep Muscles. Bone, 36, 1086-1093. https://doi.org/10.1016/j.bone.2005.02.017

[21] Anderson, A.F. and Green, N.E. (1991) Residual Functional Deficit after Partial Fibulectomy for Bone Graft. Clinical Orthopaedics and Related Research, 267, 137-140. https://doi.org/10.1097/00003086-199106000-00019

[22] Babhulkar, S.S., Pande, K.C. and Babhulkar, S. (1995) Ankle Instability after Fibular Resection. Journal of Bone and Joint Surgery British, 77, 258-261. https://doi.org/10.1302/0301-620X.77B2.7706342

[23] Burstone, M.S. (1958) Histochemical Demonstration of Acid Phosphatase with Naphthol AS-Phosphate. Journal of the Natural Cancer Institute, 21, 523-539.

[24] Amizuka, N., Yamada, M., Watanabe, J., Hoshi, K., Fukushi, M., Oda, K., Ikehara, Y. and Ozawa, H. (1998) Morphological Examination of Bone Synthesis via Direct Administration of Basic Fibroblast Growth Factor into Rat Bone Marrow. Microscopy Research and Technique, 41, 313-322.

https://doi.org/10.1002/(SICI)1097-0029(19980515)41:4<313::AID-JEMT4>3.0.CO; $\underline{2-\mathrm{R}}$

[25] Arai, E., Nakashima, H., Tsukushi, S., Shido, Y., Nishida, Y., Yamada, Y., Sugiura, H. and Katagiri, H. (2005) Regenerating the Fibula with Beta-Tricalcium Phosphate Minimizes Morbidity after Fibula Resection. Clinical Orthopaedics and Related Re- 
search, 431, 233-237. https://doi.org/10.1097/01.blo.0000146467.01032.a0

[26] Stevens, M.M., Marini, R.P., Schaefer, D., Aronson, J., Langer, R. and Shastri V.P. (2005) In Vivo Engineering of Organs: The Bone Bioreactor. Proceeding of the National Academy of Sciences of the United States of America, 102, 11450-11455. https://doi.org/10.1073/pnas.0504705102

[27] Cong, Z., Wang, J. and Zhang, X. (2000) Osteoinductivity and Biomechanics of a Porous Ceramic with Autogenic Periosteum. Journal of Biomedical Materials Research, 52, 354-359.

https://doi.org/10.1002/1097-4636(200011)52:2<354::AID-JBM15>3.0.CO;2-D

[28] Miettinen, M., Lindenmayer, A.E. and Chaubal, A. (1994) Endothelial Cell Markers CD31, CD34, and BNH9 Antibody to $\mathrm{H}$ - and Y-Antigens-Evaluation of Their Specificity and Sensitivity in the Diagnosis of Vascular Tumors and Comparison with Von Willebrand Factor. Modern Pathology, 7, 82-90.

[29] Breiteneder-Geleff, S., Soleiman, A., Kowalski, H., Horvat, R., Amann, G., Kriehuber, E., Diem, K., Weninger, W., Tschachler, E., Alitalo, K. and Kerjaschki, D. (1999) Angiosarcomas Express Mixed Endothelial Phenotypes of Blood and Lymphatic Capillaries: Podoplanin as a Specific Marker for Lymphatic Endothelium. The American Journal of Pathology, 154, 385-394.

https://doi.org/10.1016/S0002-9440(10)65285-6

[30] Edwards, J.R., Williams, K., Kindblom, L.G., Meis-Kindblom, J.M., Hogendoorn, P.C., Hughes, D., Forsyth, R.G., Jackson, D. and Athanasou, N.A. (2008) Lymphatics and Bone. Human Pathology, 39, 49-55.

https://doi.org/10.1016/j.humpath.2007.04.022 